\title{
UCRL-CONF-231936
}

LAWRENCE LIVERMORE N A T IO N A L

\section{Compact Proton Accelerator for Cancer Therapy}

Yu-Jiuan Chen, Arthur C. Paul

June 20, 2007

2007 Particle Accelerator Conference Albuquerque, NM, United States June 25, 2007 through June 29, 2007 
This document was prepared as an account of work sponsored by an agency of the United States Government. Neither the United States Government nor the University of California nor any of their employees, makes any warranty, express or implied, or assumes any legal liability or responsibility for the accuracy, completeness, or usefulness of any information, apparatus, product, or process disclosed, or represents that its use would not infringe privately owned rights. Reference herein to any specific commercial product, process, or service by trade name, trademark, manufacturer, or otherwise, does not necessarily constitute or imply its endorsement, recommendation, or favoring by the United States Government or the University of California. The views and opinions of authors expressed herein do not necessarily state or reflect those of the United States Government or the University of California, and shall not be used for advertising or product endorsement purposes. 


\title{
COMPACT PROTON ACCELERATOR FOR CANCER THERAPY*,+
}

\author{
Yu-Jiuan Chen ${ }^{\#}$ and Arthur C. Paul \\ Lawrence Livermore National Laboratory, Livermore CA 94551
}

\begin{abstract}
An investigation is being made into the feasibility of making a compact proton dielectric wall (DWA) accelerator for medical radiation treatment based on the high gradient insulation (HGI) technology. A small plasma device is used for the proton source. Using only electric focusing fields for transporting and focusing the beam on the patient, the compact DWA proton accelerator system can deliver wide and independent variable ranges of beam currents, energies and spot sizes.
\end{abstract}

\section{INTRODUCTION}

Proton therapy for cancer treatment has been considered a preferable treatment compared with the conventional radiation treatment since the location of the proton beam's Bragg peak is highly localized. By tuning the proton beam's energy profile, treatment doses can be deposited in tumors at different depths in the patient. The current proton therapy facilities have several characteristics. First, these machines typically have very large footprints, and hence they are very expensive to build. The current advance in the high gradient insulation (HGI) technology $[1,2,3,4]$ has made it possible to have a compact, short pulse DWA accelerator with an average acceleration gradient in the range of $100 \mathrm{MeV} / \mathrm{m}$. Based on this technology, a compact proton accelerator for medical radiation treatment may have only a tenth or less of the footprint, and can even be fitted in a single treatment room. Second, to allow the proton beam's Bragg peak to address tumors at different depths in the patient, the proton beam energy should be tunable between $70 \mathrm{MeV}$ and $250 \mathrm{MeV}$. Currently, tuning the beam energy is done through scattering the proton beams with masks of variable thickness on most proton therapy machines. However, large neutron production during the scattering process creates a serious health concern for patients and treatmet providers. Third, tumors come with various sizes and shapes. Currently, the typical proton therapy machines only have limited beam spot size tunability. To deposit the desired doses in the tumors while minimizing the doses in the surrounding healthy tissues, masks with various shapes are also used to shape the beam. Again, more neutrons will be created.

\footnotetext{
* Patent pending.

+ This work was performed under the auspices of the U.S. Department of Energy by University of California, Lawrence Livermore National Laboratory under Contract W-7405Eng-48.

\# yjchen@1lnl.gov
}

\section{ACCELERATOR CONFIGURATION}

We have been investigating the feasibility of making a compact, short pulse proton DWA accelerator $[1,5$. 6. 7 . $8]$ for cancer treatment by using the HGI technology. The desired radiation dose is consistent with a beam charge of 40 pico-coulombs. The desired tuning range for the beam energy is between $70 \mathrm{MeV}$ and $250 \mathrm{MeV}$, and the tuning range for the spot size is over $2 \mathrm{~mm}$ to $10 \mathrm{~mm}$.

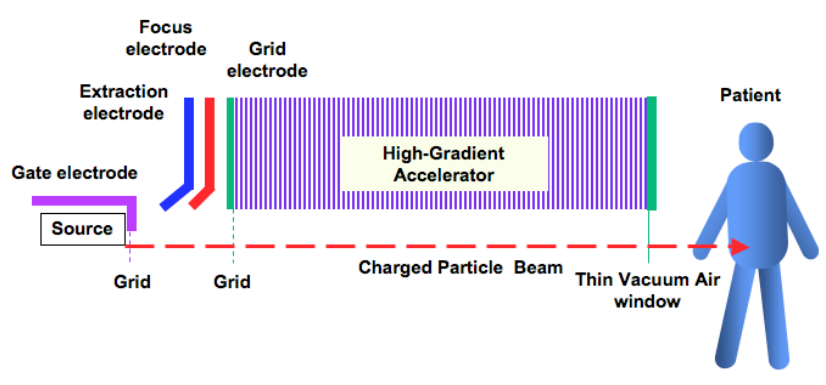

Figure 1: Schematic of the compact high-gradient DWA proton accelerator.

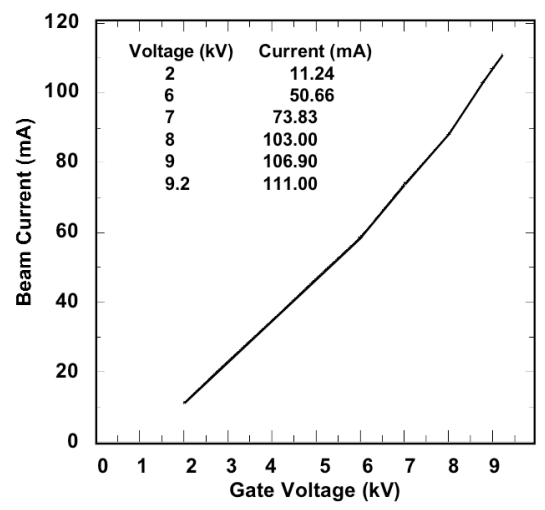

Figure 2: The extracted proton beam current as a function of the gate electrode voltage on a high-gradient proton therapy accelerator.

The schematic of the compact proton accelerator configuration is shown in Figure 1. The length of the DWA accelerator section is $2.5 \mathrm{~m}$, and the length of the injector, excluding the source and emission gap, is only $13 \mathrm{~cm}$. The particle source is a small $2 \mathrm{~mm}$ plasma device [9] from which a several nano-second pulse can be extracted. The beam current is selectable by the voltage pulses of the gate electrode. With a 2-mm emission gap, the beam current is adjustable in the range of $10-100 \mathrm{~mA}$, 
as shown in Figure 2. This beam is then accelerated and focused by the next three electrodes forming an Accelerate-Deaccelerate-Accelerate (ADA) structure leading to the dielectric wall accelerator (DWA) block. A transparent grid terminates the injector section and prevents the very high gradient of the HGI structure from influencing the overall focusing of the system. The beam energy is determined by the length of the DWA structure that is charged. This design offers independent selection of beam dose, size and energy.

\section{SPOT SIZE CONTROL}

The high-gradient compact DWA proton accelerator's injector uses a set of electrodes, consisting of an extraction electrode, a focus electrode and a grid electrode, to catch the space charge dominated beam and focus it onto the patient. The potential contour plot in the ADA structure formed by these electrodes is shown in Figure 3. The voltages on the extraction electrode, the focus electrode and the anode/grid electrode at the highgradient accelerator entrance are $980 \mathrm{kV}, 90 \mathrm{kV}$ and 980 $\mathrm{kV}$. Figure 3 shows that the voltages on the shaped extraction electrode and focus electrode make the beam going through a focusing-defocusing-focusing region, and experiencing strong net focusing forces. At the exit of the injector or the entrance of our high-gradient accelerator, there is a gird electrode to prevent the high accelerating gradient of the HGI structure from affecting the ADA structure's focusing effects. By setting the voltage on the gird electrode at the exit of the injector or the entrance of the accelerator the same as the extraction electrode voltage, the shaped focus electrode's voltage determines the final spot, which is independent of the beam current and energy.

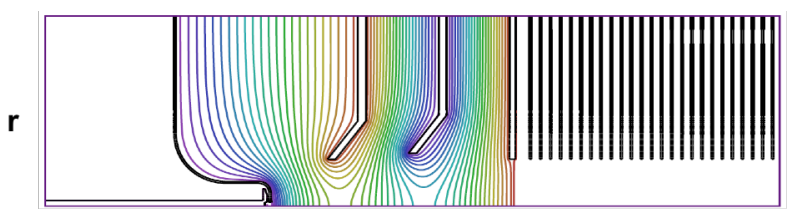

$\mathbf{z}$

Figure 3: Potential contours in a high-gradient proton accelerator.

The $r$-r' phase spaces of the 47.7-mA beam (for the gate electrode' voltage at $5 \mathrm{kV}$ ) at the location just passing the focus electrode $(\mathrm{z}=10 \mathrm{~cm})$ with various focus electrode voltage settings are given in Figure 4, showing a large range of beam divergence tunability with the voltage on the focus electrode. Note that all the simulation results presented in the paper were obtained by ignoring the short pulse and longitudinal effects. Simulation results including the longitudinal effects are presented in Ref. [5]. Figure 5 shows simulated beam transport through a magnet-free $250-\mathrm{MeV}$ proton high-gradient accelerator with various focus electrode voltage setting. The corresponding focus electrode voltages are given on the plots. The simulated beam current is $100 \mathrm{~mA}$. For these simulations, the nominal gate electrode's voltage is $9 \mathrm{kV}$, both the extraction and grid electrode are at $980 \mathrm{kV}$, and the high-gradient accelerator's acceleration gradient is $100 \mathrm{MV} / \mathrm{m}$. Figure 5 clearly shows that the spot size of the $250-\mathrm{MeV}$ and $100-\mathrm{mA}$ proton beam on the target can be easily tuned by the focus electrode voltage.

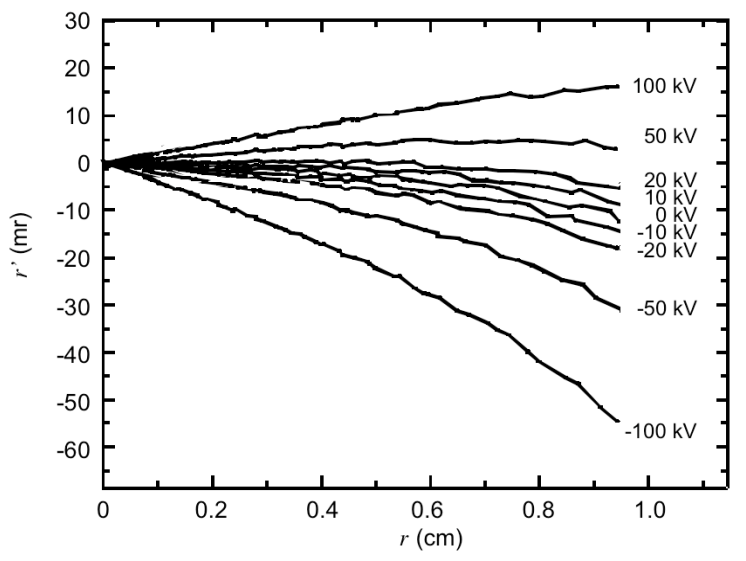

Figure 4: The $r$-r' phase spaces of the 47.7-mA beam at the location just passing the focus electrode with various focus electrode voltage settings.

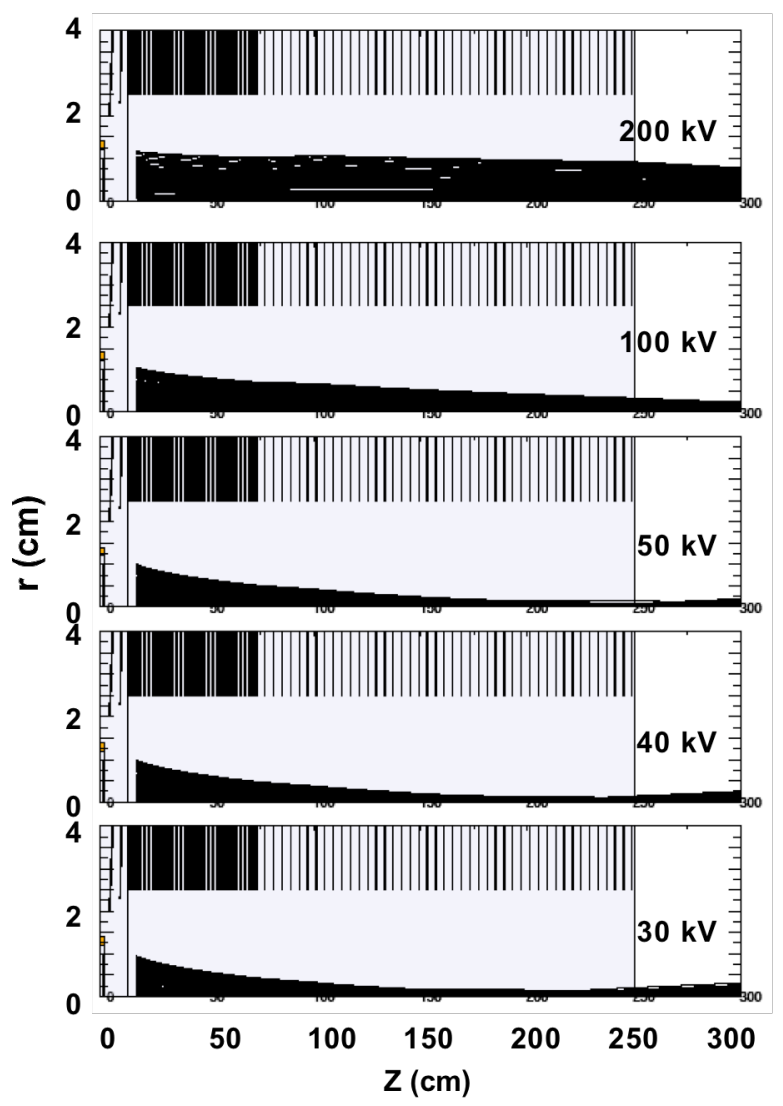

Figure 5: Beam transport in a magnet-free 250-MeV highgradient proton accelerator with various focus electrode voltage settings. 
The final beam spots of the $250-\mathrm{MeV}$ beam with various gate voltages are presented in Figure 6, which show that the final rms spot size is not sensitive to the beam current. Since the final spot size is not sensitive to the gate electrode's voltage setting, the gate voltage provides an easy control knob to turn on/off the beam current without affecting the spot size as indicated by Figure 2.
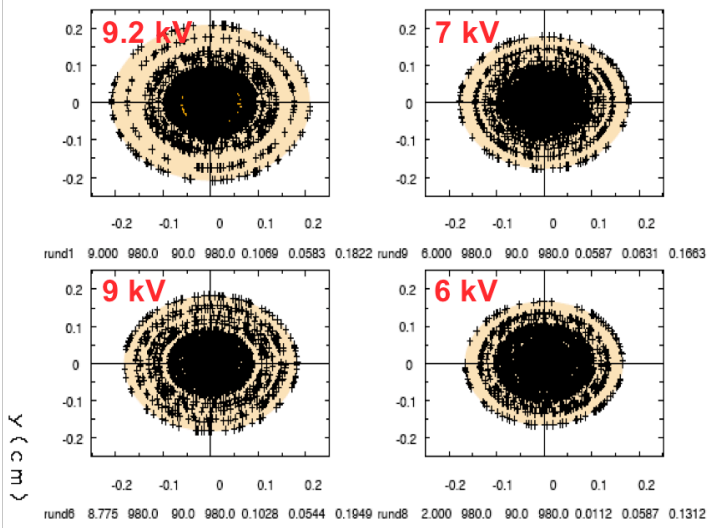

Tunde $\quad 8.775 \quad 980.0 \quad 90.0980000 .1028 \quad 0.0544$
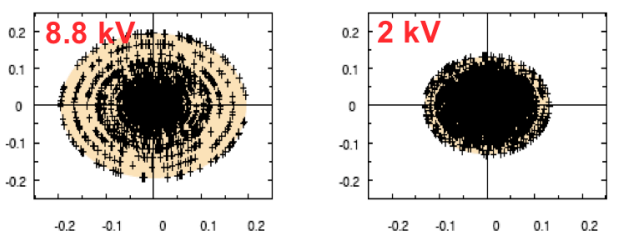

rund11 $8.000 \quad 980.0 \quad 90.0980 .00 .0882 \quad 0.0507 \quad 0.1812$

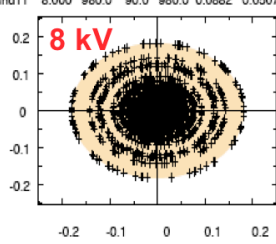

$\begin{array}{lllllll}0 & 01 & 0 & 0 & 0 & 0 & 0\end{array}$

$\mathrm{x}(\mathrm{cm})$

Figure 6: Final spot sizes of the $250-\mathrm{MeV}$ beam on the target with various gate electrode voltage.

The final beam energy is determined by the length of the DWA structure that is charged. To achieve the full $250-\mathrm{MeV}$, the entire DWA structure will be fired. To achieve the lower energy, only the front end of the DWA structure will be charged. Since beam focusing is done with the electrodes in the injector only, this strategy of tuning beam energy allows independent selection of beam size and energy.

Plots of spot sizes versus the focus electrode voltage for various proton beam energies are shown in Figure 7. Two curves are plotted for each proton energy. The upper curves present the edge radii of the beam, and the lower curves present the core radii. These plots show that a wide range of spot sizes $(2 \mathrm{~mm}-2 \mathrm{~cm}$ diameter) can be obtained for the $70-250 \mathrm{MeV}, 100-\mathrm{mA}$ proton beam by adjusting the focus electrode voltage on a high-gradient proton therapy accelerator with an accelerating gradient of 100-MV.
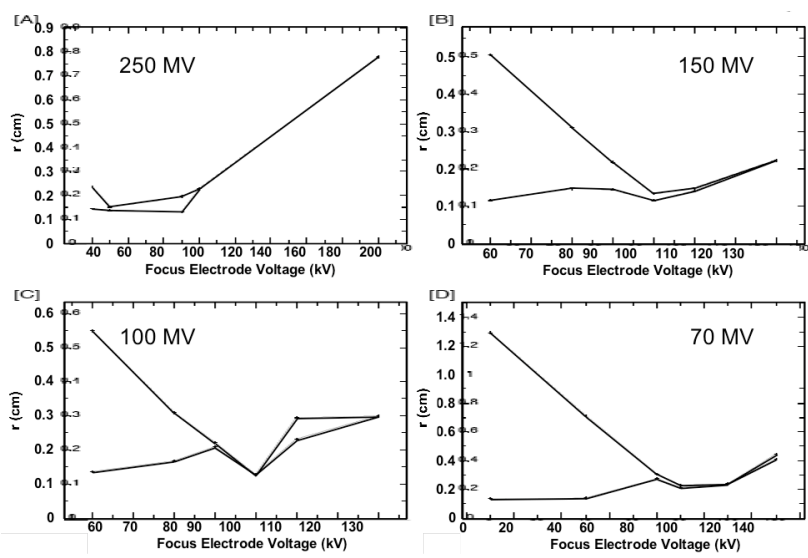

Figure 7: The edge beam radii (upper curves) and the core radii (lower curves) on the target versus the focus electrode voltage for the (a) $250-\mathrm{MeV}$, (b) $150-\mathrm{MeV}$, (c) $100-\mathrm{MeV}$ and (d) $75-\mathrm{MeV}$ proton beams. These results indicate that a large range of beam spot size can be achieved by varying the focus electrode's voltage.

\section{CONCLUSION}

In this paper, we present a conceptual layout for a compact, high-gradient DWA proton accelerator for cancer treatment. Using only electric focusing fields for transporting and focusing the beam on the patient, the compact DWA proton accelerator system should be able to deliver a wide range of beam currents, energies and spot sizes independently.

\section{REFERENCES}

[1] S.E. Sampayan et al., Proc. XVIIIth Intern. Symp. Discharges and Elect. Insul. in Vacuum, Eindhoven, The Netherlands, Aug. 17-21, 1998.

[2] G.J. Caporaso et al., "High Gradient Induction Accelerator," these proceedings.

[3] J.R. Harris et al., IEEE Trans. Dielectrics and Elect. Insul., in press.

[4] J. Harris, et al., "Vacuum Insulator Studies for the Dielectric Wall Accelerator," these proceedings.

[5] D. Blackfield, et al., "Particle Simulation of the Injector and Beam Transport in a Compact Linear Proton Accelerator," these proceedings.

[6] S.D. Nelson, et al., "Electromagnetic Simulations of Linear Proton Accelerator Structures using Dielectric Wall Accelerators," these proceedings.

[7] B.R. Poole and S.D. Nelson, "Particle Simulations of a Linear Proton Dielectric Wall Accelerator." These proceedings.

[8] L. Wang, et al., "Electromagnetic and Thermal Simulations for the Switch Region of a Compact Proton Accelerator," these proceedings.

[9] S. Sampayan, private communications. 